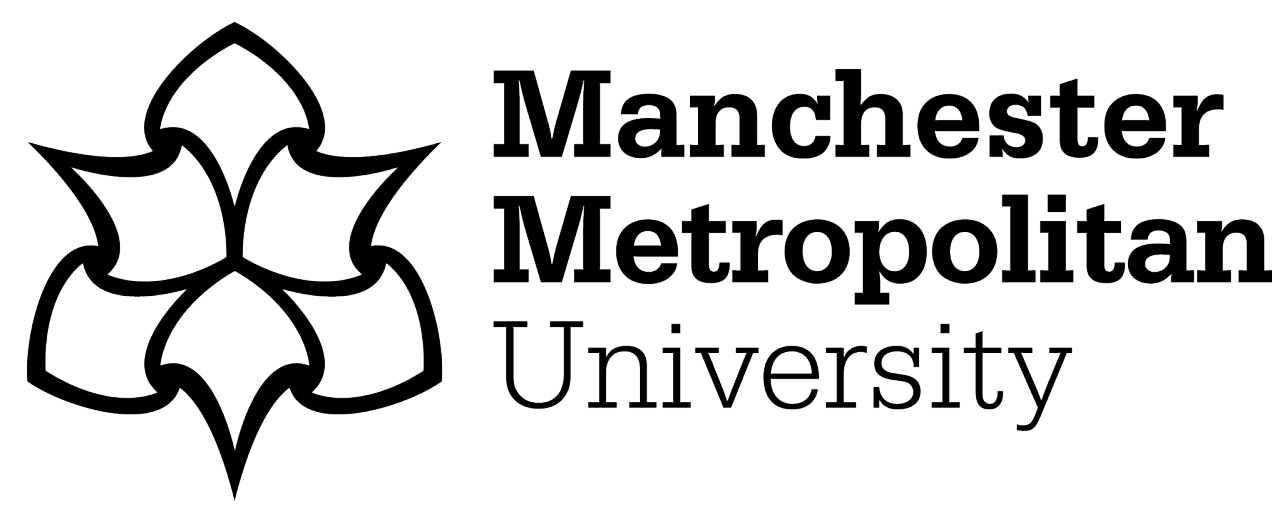

Hostler, Thomas J ORCID logoORCID: https://orcid.org/0000-0002-4658692X, Poerio, Giulia Lara and Blakey, Emma (2019) Still More Than a Feeling: Commentary on Cash et al., "Expectancy Effects in the Autonomous Sensory Meridian Response" and Recommendations for Measurement in Future ASMR Research. Multisensory Research, 32 (6). pp. 521-531. ISSN 2213-4794

Downloaded from: https://e-space.mmu.ac.uk/623072/

Version: Accepted Version

Publisher: Brill Academic Publishers

DOI: https://doi.org/10.1163/22134808-20191366

Please cite the published version 


\title{
Still More than a Feeling: Commentary on Cash et al., "Expectancy effects in the Autonomous Sensory Meridian Response" and Recommendations for Measurement in Future ASMR Research.
}

\author{
Thomas J. Hostler ${ }^{\mathrm{a} *}$, Giulia Lara Poerio ${ }^{\mathrm{b}}$ and Emma Blakey ${ }^{\mathrm{b}}$ \\ ${ }^{a}$ Department of Psychology, Manchester Metropolitan University, Manchester, UK. \\ ${ }^{b}$ Department of Psychology, University of Sheffield, Sheffield, UK.
}

\section{*Corresponding Author:}

Thomas J. Hostler

Department of Psychology

Manchester Metropolitan University

53 Bonsall St,

Manchester

M15 6GX

Tel: 01612472080

Email: t.hostler@mmu.ac.uk

\section{CONTRIBUTION STATEMENT}

T.H. and G.P. conceived of and developed the presented idea and contributed equally. T.H., G.P., \& E.B. all contributed to the content and writing of the manuscript.

\section{Summary:}

Autonomous Sensory Meridian Response (ASMR) - the sensory phenomenon experienced by some people in response to visual and auditory stimuli such as whispering - has attracted substantial public attention but is not yet well-understood or well-established within the scientific community. Recent research published in PeerJ by Cash, Heisick, \& Papesh (2018) investigated whether ASMR could be a placebo effect (resulting from expectation) rather than a genuine experience triggered by ASMRinducing stimuli. In this article, we provide a commentary on Cash et al.'s findings and argue that they provide evidence for (rather than against) the veracity of ASMR. We discuss issues regarding measurement of ASMR and end by providing some recommendations on how to assess ASMR as both a state and a trait, in the hope of galvanising collaborative research efforts in the emerging field of ASMR.

Key words: ASMR, Autonomous Sensory Meridian Response, Placebo effect, Expectation effect, Commentary

Short Title: Commentary on Cash et al. 


\section{Still more than a feeling: Commentary on Cash et al., "Expectancy effects in the Autonomous Sensory Meridian Response" and recommendations for measurement in future ASMR research.}

This article is a commentary on: Cash, D. K., Heiseck, L. L., \& Papesh, M. H. (2018) Expectancy effects in the Autonomous Sensory Meridian Response. PeerJ, 6:e5229, doi: http://10.7717/peerj.5229

\section{Introduction}

Autonomous Sensory Meridian Response (ASMR) is a sensory experience characterised by involuntary tactile tingling sensations originating in the scalp which are accompanied by feelings of relaxation (Poerio et al., 2018). The sensation occurs in some people in response to audio, visual, and tactile triggers which commonly include: whispering, close personal attention, tapping, and slow hand movements (Barratt and Davis, 2015, Barratt et al., 2017, Fredborg et al., 2017, Poerio et al., 2018). Although people with ASMR report experiencing the feeling since childhood (Poerio, 2016), public awareness of the phenomenon has burgeoned in recent years due to the proliferation of online ASMR videos created to induce the sensation 'on demand' (Poerio, 2016). Many ASMR experiencers report using these videos as a sleep-aid and to alleviate symptoms of anxiety and depression (Barratt and Davis, 2015). Despite this, there are currently very few published scientific studies which examine ASMR, its veracity, and potential therapeutic benefits.

\section{Is ASMR a real or placebo effect?}

In a recent article published in PeerJ, Cash et al. (2018), sought to determine "whether it [ASMR] truly exists, or rather is a product of individual expectations" and "may represent a placebo effect" (p.3). Specifically, the aim of their study was "to assess whether ASMR is affected by individuals' expectations or if the phenomenon emerges regardless of expectations." (p. 4). This is a timely and important question given the emergence of ASMR research: If ASMR can be explained entirely by expectation, then future costly and timeconsuming research into biological causes of ASMR could be unwarranted or even unethical (Boot et al., 2013). 
Cash et al. tested two groups of participants: ASMR participants who experience the sensation (recruited from ASMR discussion boards), and naïve non-ASMR participants who do not (a post-experimental assessment showed they had no previous exposure to ASMR clips). Participants were presented with audio clips featuring: 1) ASMR content from YouTube including several common triggers; 2) perceptually similar "foils" or control clips (e.g., finger drumming), and 3) a control music clip. Participants rated the extent to which they experienced ASMR after each clip from 1 (not at all) to 7 (a great deal). The experience of ASMR was described to participants as "a pleasant tingling, static-like sensation in response to listening to specific audio or visual stimuli” (p. 6). The key experimental manipulation designed to test the role of expectancy effects was the instruction given to participants before listening to the clips. In the encouraging condition, participants were told that all of the clips had been shown to induce ASMR, and the experiment sought to determine the 'causal mechanisms' of the effect; in the discouraging condition, participants were told that none of the clips had been shown to induce ASMR, and that the purpose of the experiment was to determine audio-visual characteristics that prevent ASMR from occurring.

The results: Naïve, but not ASMR participants are susceptible to the expectancy manipulation

Cash et al.'s primary finding was that naïve non-ASMR participants were susceptible to the expectancy manipulation, but ASMR participants were not. Naïve participants reported significantly higher ASMR ratings when they were encouraged, rather than discouraged, to think that the clips induced ASMR. However, ASMR participants were immune to the expectation manipulation: their ASMR ratings did not differ depending on whether they were encouraged or discouraged to think that the clips induced ASMR. Further analyses showed that ASMR participants, but not naïve participants, had significantly different ratings for different clip types (irrespective of the manipulation): ASMR participants rated ASMR clips as significantly more ASMR-inducing compared to both the foil and music clips whereas naïve participants' ratings were similar for all clip types. This overall pattern of responses is represented in Fig. 1.

[Figure 1 here] 
Figure 1. ASMR and non-ASMR participants mean ASMR ratings of all types of clips, for both encouraging and discouraging instructions. Error bars represent Standard Errors of the Mean. This figure was created in $R$ from raw data provided by Cash et al. Code to reproduce this graph is available on the OSF: https://osf.io/axn3d/

\section{Is ASMR a placebo effect?}

Cash et al. interpret the observed Group (ASMR vs. naïve participant) X Instruction (encouraging vs. discouraging) interaction: "we found that ASMR users were immune to our expectation manipulation, but naïve users experienced ASMR when they were told to expect it and did not experience ASMR when told not to expect it” (p.4). However, their overall conclusion in the discussion was that "[the] findings support expectancy effects from both experienced ASMR users and naïve participants" (p.12). This conclusion is based on the Clip (ASMR vs. foil vs. music) X Group (ASMR vs. naïve participant) interaction where ASMR participants rated foil and music clips as less ASMR-inducing than naïve participants, regardless of instruction. They interpret these findings as showing that "[ASMR participants] were affected by their own expectations, driven by their history of ASMR viewing or participation in ASMR discussion boards" (p.11). The suggestion here is that ASMR participants were affected by their own expectations to report reduced ASMR to non-ASMR clips, implying that their personal expectations overrode the experimentally induced expectancy manipulation: "ASMR users either recognized the ASMR clips, or were familiar enough with the characteristics of "real" ASMR media to report effects consistent with their expectations (i.e., intentionally reporting lower ASMR ratings).” (p.11).

\section{An alternative explanation}

Examining the entire set of observed results, we diverge from Cash et al.'s conclusions from the experiment. Rather than demonstrating that ASMR occurs from expectancy (induced or pre-existing), our interpretation is that their experiment provides convincing evidence for the veracity of ASMR: it is not a placebo effect but genuinely triggered in people who experience it. They showed that people who experience ASMR report the ASMR sensation in response to ASMR clips regardless of any expectancy manipulation. When ASMR participants were misdirected to think that ASMR clips prevent ASMR, they still consistently reported the clips as ASMR inducing; and when they were misdirected to think that the control clips were likely to trigger ASMR, they still consistently reported them as less triggering than actual ASMR clips. This evidence supports the view that ASMR is a genuine experience and is 
inconsistent with the view that it emerges to any ostensibly 'ASMR' stimuli because of expectation.

Our interpretation is also in line with Cash et al.'s findings that ASMR participants rated foil and control music clips lower than non-ASMR participants, regardless of instruction. Cash et al.'s interpretation of these results (i.e., that they represent evidence that ASMR participants are deliberately rating these clips as less ASMR inducing due to expectation) is logically problematic. Low ASMR ratings of non-ASMR clips cannot explain whether high ASMR ratings of ASMR clips are caused by expectation or not. Even if they could, the most parsimonious explanation is that the foil and music clips did not physically cause ASMR in the ASMR participants, and thus were accurately rated as low. ASMR participants have the perceptual expertise required to identify the ASMR sensation (both in terms of the physical tingling sensations and associated emotional responses), and were therefore able to accurately report whether or not they experienced ASMR to a video during the study. In contrast, naïve non-ASMR participants do not have the perceptual expertise required to correctly classify any sensations experienced in response to the videos as 'ASMR' or something else. Therefore "ASMR-ratings" from naïve participants were not accurate representations of how ASMRinducing the media was and may not be considered valid reports of ASMR.

Indeed, Cash et al. acknowledge that non-ASMR participants may have actually been experiencing frisson, or music chills, when rating the music clip as inducing ASMR. Without further questioning of participants we are unable to tell whether the tingles were ASMR, frisson, goosebumps, or even just a more general physical feeling of relaxation (del Campo and Kehle, 2016, Maruskin et al., 2012). Cash et al. acknowledge that the phenomenological difference between frisson and ASMR was reported to them by the ASMR participants and the difference is promoted within the ASMR reddit community from which they sampled (https://www.reddit.com/r/asmr/wiki/index).

Overall, Cash et al.'s results provide important evidence for the veracity of ASMR in those that claim to experience it and demonstrate that expectation is unlikely to be the cause of reported ASMR tingling in experiencers. Their findings are consistent with a small but growing body of ASMR research demonstrating that there are physiological and neurological differences between ASMR experiencers and non-experiencers. Our own studies have compared the emotional and physiological responses of ASMR and non-ASMR participants in response to both ASMR and non-ASMR video clips (Poerio et al., 2018). Similar to Cash 
et al., we found that only ASMR participants reported tingling sensations in response to ASMR videos (but not control videos). We also identified consistently different physiological and self-reported emotional responses between ASMR and non-ASMR participants in response to ASMR videos (e.g., greater increases in calmness/relaxation and greater reductions in heart rate). Smith et al. (2017) have demonstrated differences between ASMR and non-ASMR participants in terms of their resting state brain activity. Specifically, ASMR participants, compared to non-ASMR participants, showed reduced functional connectivity between frontal, sensory and attentional regions of Default Mode Network. Smith et al. (2017) suggest that these differences may reflect the inability of ASMR participants to inhibit sensory-emotional experiences (such those induced by ASMR triggers).

\section{Moving forward: recommendations for ASMR research}

We believe that Cash et al.'s interpretations may stem partly from a lack of clarity concerning the characterisation and measurement of ASMR which is both (1) a state (the emotional experience of ASMR in real time - i.e., feeling ASMR) and (2) a trait (whether an individual reliably experiences ASMR or not - i.e., having ASMR). Being able to accurately measure the ASMR state and discriminate between those who have the ASMR trait are two key methodological issues for future ASMR research, and essential for robustly examining whether ASMR is a result of participant demand or expectation. Here we provide a set of recommendations to improve efforts in the field for laboratory measurement (state ASMR) and participant recruitment (trait ASMR).

\section{The ASMR state: Measuring the presence/absence/degree of ASMR}

Future ASMR research is likely to require the accurate measurement of the ASMR state, and the degree to which it is experienced (for example, this would allow the examination of individual differences within an ASMR sample - such as a comparison of those with more intense vs. mild ASMR experiences). Although we have demonstrated that the ASMR state is associated with physiological changes (Poerio et al., 2018), the routine use of physiological measures to determine/corroborate whether a person is experiencing the ASMR state is likely to be practically prohibitive (e.g., in online studies). We suggest that when attempting to assess the ASMR state via Likert-scale measures after viewing ASMR content, multiple measures should be used, in order to: (1) accurately establish that the sensations felt are 
characteristic of ASMR and (2) to rule out the possibility that experienced sensations reflect another type of chill sensation. We have detailed these in Table 1.

\section{[Insert Table 1 here]}

We also recommend assessing how the ASMR state is experienced under research conditions compared to participants' everyday lives. Our previous research shows that the ASMR state is experienced less intensely in the laboratory compared to in everyday life (e.g., when watching ASMR content at home) (Poerio et al., 2018). The question we used to measure this was: "Compared to how you usually experience ASMR (e.g., watching videos at home or in daily life) how was your experience of ASMR during this study?" $(1=$ much less intense, $2=$ less intense, 3 = about the same, $4=$ more intense, $5=$ much more intense).

\section{The ASMR trait: establishing ASMR status for participant selection}

Future ASMR research will inevitably rely on recruiting participants with ASMR and some studies will also seek to compare ASMR experiencers with non-experiencers. Currently, it is unclear whether trait ASMR is a binary phenomenon or occurs on a continuum. In our experience, participants are generally likely to self-select as either an ASMR or non-ASMR participant, but within the category of 'ASMR participants' there is variability in the intensity of ASMR experiences (e.g., some may have particularly intense ASMR experiences whereas others feel the sensation more mildly). Nevertheless, future research will likely require a control group of individuals who do not experience ASMR (to any degree) for comparative purposes. We recommend that trait ASMR should be independently verified prior to investigation using a standardised protocol which we describe below. We suggest steps 1-3 as a minimum for the recruitment of ASMR/control participants.

1. Description of ASMR. Participants should be provided with a detailed description of ASMR (including emotional correlates, common triggers) and information on the distinction between ASMR and other sensations (e.g., music induced chills). They should be asked, based on the description, whether they experience ASMR or not (yes, no, not sure).

2. ASMR checklist. Participants should complete a checklist/questionnaire that records other features characteristic of the ASMR trait (from previous research)- for example: age of first ASMR experience, a personal triggers checklist (including foil triggers), emotional responses, a written description of the experience and location and direction of tingles on the body. For an example checklist see Fredborg et al. (2017). 
3. ASMR videos. Participants should watch several ASMR videos to confirm ASMR vs. non-ASMR status as per Smith et al. (2017) and Fredborg et al. (2017). This is likely to be particularly important for recruiting non-ASMR participants: in these studies, non-ASMR participants who reported any tingles in response to an ASMR video were excluded as control participants.

4. Consistency tests. Although they are labour-intensive, it is possible that consistency tests for ASMR participants would help to establish trait ASMR (i.e., whether ASMR is consistently experienced to triggers over time). Research on the related sensory phenomenon of synaesthesia has employed such tests in which participants describe their synesthetic experience in response to stimuli on different occasions (e.g., separated by a week). Synaesthesia status is determined by the consistency of the reports such that synesthetes report more consistent responses to stimuli, even compared to controls instructed to fake a synesthetic response (Eagleman et al., 2007). A similar consistency test could present ASMR/control participants with different ASMR and non-ASMR inducing stimuli on multiple occasions to examine consistency in ASMR responses over time. Eagleman et al. (2007) have developed a website (http://www.synesthete.org) hosting a battery of consistency tests as a resource for researchers to determine synaesthesia status in potential participants prior to experimental work, and a similar standardised resource for recruitment of ASMR participants would be a valuable contribution to the field. However, we should note that the use of consistency tests for distinguishing ASMR responders from nonresponders may rest on assumptions regarding what 'counts' as experiencing ASMR that have yet to be verified ${ }^{1}$. This issue is highlighted by Simner (2011) within the context of consistency tests for synaesthesia: classification based on consistent synesthetic responses to the same stimuli may be too narrow because it would exclude genuine synesthetes who experience strong cross-sensory pairings (e.g., seeing words as colours) albeit not consistently. The same issue may be relevant for classifying trait ASMR. If a person who claims to experience ASMR does not always experience the sensation in response to the same specific stimulus (e.g., soft speaking) over time, then would it be correct to classify this individual as a non-responder? We suspect not because the experience of ASMR is likely to be idiosyncratic and dependent on other contextual factors. This issue is particularly pertinent given the anecdotal reports of

\footnotetext{
${ }^{1}$ We thank an anonymous reviewer for bringing this point to our attention.
} 
“ASMR Immunity" (Ahuja, 2016) which refers to ASMR experiencers developing a habituation to ASMR stimuli, meaning that they are less likely to experience the sensation after over-exposure to ASMR media. Therefore, while consistency tests may be a useful addition for participant recruitment, they may be too narrow in and of themselves to characterise ASMR as a trait. On the one hand consistency tests would probably exclude many people who we would conclude from other measures (such as questionnaires) as having the ASMR trait. However, on the other hand, consistency tests might identify the strongest ASMR responders which would be useful for research when examining any potential differences between those who experience ASMR and those that do not (particularly for time and cost intensive research such as brain imaging).

\section{Conclusion}

We welcome Cash et al.'s study which we have argued provides evidence for the veracity of ASMR and contributes to the exciting emerging field of ASMR research. We hope to have made some useful methodological recommendations for those conducting or considering conducting research in the field at this early stage. A consensus on the criteria for establishing and measuring the ASMR trait and state are important prerequisites for progressing the field and allowing the comparison of research studies in the future. Indeed, consistency of measurement is an important issue in psychological research, and fields in which there are multiple commonly-used measures have often suffered from biased and inconsistent results which hampers progress (e.g., depression research, Fried, 2017).

We hope that our recommendations represent a strong foundation for researchers in the field to work together to establish a defined and accepted protocol for measuring ASMR so that we can better understand the fascinating phenomenon of ASMR.

\section{Acknowledgements}

We would like to thank Brendan O'Connor for his comments on an early draft as well as Oliver Clark for his help producing Figure 1. 
Table 1. Criteria to use when measuring state ASMR using questionnaire measures.

\begin{tabular}{|c|c|c|}
\hline ASMR measure & Explanation & Example items \\
\hline $\begin{array}{l}\text { Frequency and } \\
\text { time course of } \\
\text { tingling sensations }\end{array}$ & $\begin{array}{l}\text { ASMR tingling sensations are likely } \\
\text { to be of longer duration and more } \\
\text { "dynamic" in comparison to music } \\
\text { induced chills (Fredborg et al., } \\
2017 \text {, del Campo and Kehle, 2016) }\end{array}$ & $\begin{array}{l}\text { "How frequently (if at all) did you experience } \\
\text { tingling sensations during the video?" 1(none of } \\
\text { the time) to } 7 \text { (all of the time). } \\
\text { "When you experienced tingling sensations, } \\
\text { how long did they last for?" (Open ended } \\
\text { response) } \\
\text { "Which option best describes how the tingling } \\
\text { sensations felt to you?" They were sustained } \\
\text { throughout the whole clip/ They were constant } \\
\text { but came in waves as the intensity ebbed and } \\
\text { flowed/They were fleeting sensations that came } \\
\text { and went/ Other (please describe). }\end{array}$ \\
\hline $\begin{array}{l}\text { Intensity of } \\
\text { tingling sensations }\end{array}$ & $\begin{array}{l}\text { To determine intensity of ASMR } \\
\text { response (which may be useful for } \\
\text { individual difference examinations) }\end{array}$ & $\begin{array}{l}\text { "How intense were the tingling } \\
\text { sensations?"1(very mild) to } 7 \text { (very intense) }\end{array}$ \\
\hline $\begin{array}{l}\text { Location of } \\
\text { tingling sensations }\end{array}$ & $\begin{array}{l}\text { Barratt and Davis (2015) have } \\
\text { shown that ASMR tingles generally } \\
\text { start at the top of the head and move } \\
\text { down the back and shoulders, thus } \\
\text { differentiating them from other } \\
\text { kinds of chill sensations such as } \\
\text { frisson that may start on the arms or } \\
\text { shoulders (Craig, 2005). }\end{array}$ & $\begin{array}{l}\text { Present a diagram of the human body where } \\
\text { participants can indicate areas in which they } \\
\text { experienced tingles and their direction of travel, } \\
\text { and/or free text response box. }\end{array}$ \\
\hline $\begin{array}{l}\text { Emotional } \\
\text { responses }\end{array}$ & $\begin{array}{l}\text { Poerio et al. (2018) have shown that } \\
\text { ASMR tingles are consistently } \\
\text { accompanied by increased high and } \\
\text { low arousal pleasant affect (e.g., } \\
\text { feelings of excitement and } \\
\text { calmness) which differentiates them } \\
\text { from music-induced chills which are } \\
\text { typically associated more with high } \\
\text { activation pleasant affect and strong } \\
\text { emotions of being moved or } \\
\text { touched (Bannister, 2018, del } \\
\text { Campo and Kehle, 2016, Grewe et } \\
\text { al., 2009). }\end{array}$ & $\begin{array}{l}\text { "Please indicate how you feel now compared to } \\
\text { before you watched the video, from 1(much } \\
\text { less) to } 7 \text { (much more)" for various items } \\
\text { measuring high and low arousal positive and } \\
\text { negative affect (i.e., excitement, sadness, } \\
\text { calmness, and stress). Alternatively, these can } \\
\text { be calculated from pre- and post- measures. } \\
\text { Items should be taken from validated affect } \\
\text { measures indexing the four poles of the } \\
\text { circumplex model of affect (Remington et al., } \\
\text { 2000). }\end{array}$ \\
\hline
\end{tabular}




\section{References}

Ahuja, N. K. 2016. Why are people losing the blissful tingles of ASMR? [online] Available at: http://nautil.us/blog/why-are-people-losing-the-blissful-tingles-of-asmr [Accessed 18th March 2019]

Bannister, S. 2018. A Survey into the Experience of Musically induced Chills: Emotions, Situations and Music. Psychology of Music. doi:10.1177/0305735618798024

Barratt, E. L. \& Davis, N. J. 2015. Autonomous Sensory Meridian Response (ASMR): a flow-like mental state. Peerj, 3. doi:10.7717/peerj.851

Barratt, E. L., Spence, C. \& Davis, N. J. 2017. Sensory determinants of the autonomous sensory meridian response (ASMR): understanding the triggers. Peerj, 5. doi:10.7717/peerj.3846

Boot, W. R., Simons, D. J., Stothart, C. \& Stutts, C. 2013. The Pervasive Problem With Placebos in Psychology: Why Active Control Groups Are Not Sufficient to Rule Out Placebo Effects. Perspectives on Psychological Science, 8, 445-454. doi:10.1177/1745691613491271

Cash, D. K., Heisick, L. L. \& Papesh, M. H. 2018. Expectancy effects in the Autonomous Sensory Meridian Response. Peerj, 6. doi:10.7717/peerj.5229

Craig, D. G. 2005. An exploratory study of physiological changes during "chills" induced by music. Musicae Scientiae, 9, 273-287. doi:10.1177/102986490500900207

del Campo, M. A. \& Kehle, T. J. 2016. Autonomous Sensory Meridian Response (ASMR) and Frisson: Mindfully Induced Sensory Phenomenon that Promote Happiness. International Journal of School \& Educational Psychology, 4, 99-105. doi:10.1080/21683603.2016.1130582

Eagleman, D. M., Kagan, A. D., Nelson, S. S., Sagaram, D. \& Sarma, A. K. 2007. A standardized test battery for the study of synesthesia. Journal of Neuroscience Methods, 159, 139-145. doi:10.1016/j.jneumeth.2006.07.012

Fredborg, B., Clark, J. \& Smith, S. D. 2017. An Examination of Personality Traits Associated with Autonomous Sensory Meridian Response (ASMR). Frontiers in Psychology, 8. doi:10.3389/fpsyg.2017.00247

Fried, E. I. 2017. The 52 symptoms of major depression: Lack of content overlap among seven common depression scales. Journal of Affective Disorders, 208, 191-197. doi:10.1016/j.jad.2016.10.019

Grewe, O., Kopiez, R. \& Altenmuller, E. 2009. The Chill Parameter: Goose Bumps and Shivers as Promising Measures in Emotion Research. Music Perception, 27, 61-74. doi:10.1525/Mp.2009.27.1.61

Maruskin, L. A., Thrash, T. M. \& Elliot, A. J. 2012. The Chills as a Psychological Construct: Content Universe, Factor Structure, Affective Composition, Elicitors, Trait Antecedents, and 
Consequences. Journal of Personality and Social Psychology, 103, 135-157.

doi:10.1037/a0028117

Poerio, G. L. 2016. Could insomnia be relieved with a YouTube video? The relaxation and calm of Autonomous Sensory Meridian Response (ASMR). In: Callard, F., Staines, K. \& Wilkes, J. (Eds.) The Restless Compendium: Interdisciplinary investigations of rest and its opposites. Palgrave Macmillan.

Poerio, G. L., Blakey, E., Hostler, T. J. \& Veltri, T. 2018. More than a feeling: Autonomous sensory meridian response (ASMR) is characterized by reliable changes in affect and physiology. PloS One, 13. doi:10.1371/journal.pone.0196645

Remington, N. A., Fabrigar, L. R. \& Visser, P. S. 2000. Reexamining the circumplex model of affect. Journal of Personality and Social Psychology, 79, 286-300. doi:10.1037//0022-3514.79.2.286

Simner, J. 2012. Defining synaesthesisa: A response to two excellent commentaries. British Journal of Psychology, 103, 24-27. doi:10.1111/j.2044-8295.2011.02059.x

Smith, S. D., Fredborg, B. K. \& Kornelsen, J. 2017. An examination of the default mode network in individuals with autonomous sensory meridian response (ASMR). Social Neuroscience, 12, 361-365. doi:10.1080/17470919.2016.1188851 